\title{
Effect of direct bubble-bubble interactions on linear-wave propagation in bubbly liquids
}

\author{
D. Fuster, ${ }^{1,2, *}$ J. M. Conoir, ${ }^{1,2}$ and T. Colonius ${ }^{3}$ \\ ${ }^{1}$ Sorbonne Universités, UPMC Univ Paris 06, UMR 7190, Institut Jean Le Rond d'Alembert, F-75005, Paris, France \\ ${ }^{2}$ CNRS, UMR 7190, Institut Jean Le Rond d'Alembert, F-75005, Paris, France \\ ${ }^{3}$ Division of Engineering and Applied Science, California Institute of Technology, Pasadena, California 91125, USA
}

(Received 3 April 2014; published 11 December 2014)

\begin{abstract}
We study the influence of bubble-bubble interactions on the propagation of linear acoustic waves in bubbly liquids. Using the full model proposed by Fuster and Colonius [J. Fluid Mech. 688, 253 (2011)], numerical simulations reveal that direct bubble-bubble interactions have an appreciable effect for frequencies above the natural resonance frequency of the average size bubble. Based on the new results, a modification of the classical wave propagation theory is proposed. The results obtained are in good agreement with previously reported experimental data where the classical linear theory systematically overpredicts the effective attenuation and phase velocity.
\end{abstract}

DOI: 10.1103/PhysRevE.90.063010

PACS number(s): 47.55.dd, 43.35.-c, 43.30.-k, 43.25.Yw

\section{INTRODUCTION}

Acoustic wave propagation in bubbly liquids is relevant to diverse applications including cavitation reloading of surfaces exposed to underwater explosions, shockwave lithotripsy, and high-intensity focused ultrasound. Since Van Wijngaarden [1] proposed the first equations of motion for a dilute dispersion of gas bubbles in a liquid, several authors have proposed extensions to the original equations. Ainslie and Leighton [2] provide an account of this evolution of the theory of linear wave propagation in bubbly liquids. Most approaches are devoted to deriving models that account for the different types of dissipation present in the system, from the initial works of Chapman and Prosperetti [3,4] to most recent studies [5]. One of the strongest assumptions in these models is that mutual interactions among bubbles are negligible except through their effect on the mixture-averaged flow. For instance, Sangani [6] proposes correction terms for large void fractions that show that these terms tend to decrease the attenuation near the bubble resonance frequency. Neglecting bubble-bubble interactions formally restricts the applicability of the classical linear theory to low concentrations, where bubbles are so far from each other that the near field pressure around a bubble is not felt by the surrounding bubbles [7]. The averaged interspace distance between bubbles, $\bar{d}_{i j}$, is expected to scale as $\frac{\bar{d}_{i j}}{R_{0}} \approx C \beta^{-1 / 3}$, where $C$ is a constant of order unit and $\beta$ is the void fraction. For void fractions as low as $10^{-4}-10^{-3} \mathrm{vol} / \mathrm{vol}$ the averaged interspace distance is only around ten times the averaged bubble radius $R_{0}$. In the simplest analysis, we would expect the response of each bubble to be influenced by the instantaneous response of the surrounding bubbles for higher void fractions.

It is possible to find in the literature various experimental works measuring the phase velocity and attenuation of bubbly liquids [8-12]. In most of these works, the comparison of the classical linear theory and the experimental results is not satisfactory for frequencies near resonance when comparing both attenuation and phase velocity. In general, the experimental investigation of direct bubble-bubble interactions is difficult due to the presence of reverberation $[13,14]$ and the

*Corresponding author: fuster@dalembert.upmc.fr dearth of diagnostics capable of directly measuring the void fraction and bubble radius distribution [15]. As an alternative one can resort to numerical models where the value of all these variables is given. In this case, the main problem is that it is not computationally tractable, for large numbers of bubbles, to directly resolve both phases. Various volume and phase-averaged continuum approaches have been proposed in the literature. In the most sophisticated of these models, twoway coupled (dynamic) effects of cavitation are considered meaning that the time evolution of spherical bubbles are determined by solving a Rayleigh-Plesset-type (RP) equation whose driving pressure is determined from the local pressure of the continuous phase, and whose result affects the continuous phase by altering the void fraction [1,7]. Various extensions of the RP model adequately account for phase change [16], liquid compressibility, and heat and mass transfer within the bubble contents [17]. However, existing phase and volume-averaging approaches require two fundamental assumptions that limit their applicability: low void fraction, and scale separation between the typical bubble size and the length scales associated with the (averaged) flow field. In this paper, we consider a new bubbly flow methodology based on volume-averaged equations that relaxes the scale-separation assumption and directly accounts for bubble-bubble interactions [18]. The numerical results are used here to gain insight into the effect of direct bubble-bubble interactions and to propose correction terms in the classical theory for linear wave propagation in bubbly liquids in order to improve the accuracy of the predictions for frequencies near the bubble natural resonance frequency.

\section{MODEL AND NUMERICAL METHOD}

The full equations for the model are detailed in Fuster and Colonius [18]. The model utilizes a volume-averaging approach [1]. We solve for the averaged continuity, momentum and energy equations in addition to the advection equation for every component present in the system. These equations can be written as [19]

$$
\frac{\partial \rho}{\partial t}+\nabla \cdot(\rho \boldsymbol{u})=0
$$




$$
\begin{aligned}
& \frac{\partial \rho \boldsymbol{u}}{\partial t}+\nabla \cdot(\rho \boldsymbol{u} \boldsymbol{u}+p I)=0, \\
& \frac{\partial \rho e}{\partial t}+\nabla \cdot[\boldsymbol{u}(\rho e+p)]=0
\end{aligned}
$$

where $\rho$ is the averaged density, $t$ is time, $\boldsymbol{u}$ is the averaged mixture velocity, $p$ is the pressure, and $e$ is the specific energy. This set of equations is closed by specifying an equation of state. In this case we assume that this equation obeys the following form:

$$
p \Gamma+\Pi_{\infty}=\rho e-\frac{1}{2} \rho\|\boldsymbol{u}\|^{2} .
$$

In this work we consider a single bulk component (i.e., water) and a disperse phase (e.g., air bubbles) with void fraction $\beta$. This model differs from previous models in how the void fraction, $\beta$, is defined in terms of the bubble radius, and how the average pressure in a computational cell relates to the pressure felt by the corresponding bubbles in that cell. Bubbles are treated as point particles that are advected with the flow. If a polydisperse mixture is desired, then we set the initial radii of each bubble according to a representative probability density function. To compute $\beta$, we multiply each individual bubble volume by a discrete delta function that smears its value onto the computational grid and, at each point in the domain, sum this over all bubbles. To treat the dynamics of each bubble, the model introduces a new RP-like equation that accounts for a cluster of neighboring bubbles (i.e., within a computational cell). This equation is a generalization of the incompressible bubble-cluster model of Ilinskii et al. [20] to compressible liquids, and likewise assumes spherical bubbles and potential flow in the vicinity of the bubbles.

The key step in the derivation is to represent the pressure at infinity as seen by the bubbles in the potential flow model in terms of the average pressure of the liquid within a computational cell. From the potential flow solution for the bubble cluster, we may expand the pressure at infinity in terms of the cell pressure plus a correction term that can be estimated based on the bubble dynamics. The correction term thus represents a modification to the local value of pressure associated with the bubble dynamics. If the void fraction is sufficiently small, this term also becomes small and the equations relax to the classical volume-averaged equations. By taking a cell-based approach rather than a multiple-scales approach, the model we obtain is grid dependent, much in the same way a large eddy simulation (LES) of turbulence depends on the grid spacing. In the limit of the grid spacing going to zero, the LES model switches off resulting in a direct numerical simulation of the turbulent flow. In the present model, as the grid is refined to the size of an individual bubble, the model exactly represents the dynamics of an isolated spherical bubble. This is verified in detail by comparing against theoretical solutions for this case [18]. At the other extreme, the model is demonstrated to converge to the solution to ensemble-averaged models for low spatial resolution. The computational expense is greatly reduced compared to the corresponding ensemble-averaged approach. Therefore, the current model seems suitable to numerically investigate complex problems where the computational effort of both direct interface capturing and current ensemble-averaged models are prohibitive.

This cavitation model has been incorporated into a multifluid, compressible flow solver that utilizes advanced weighted essentially nonoscillatory (WENO) shock- and interfacecapturing techniques [19]. Details about the implementation of the model on the fluid solved can be found in Ref. [21].

\section{LINEAR MODELS}

\section{A. Classical linear model}

The nonlinear model presented above places no restriction on the amplitude of waves propagating through, and interacting with, the bubbles. However, for practical purposes it is interesting to derive linearized solutions for wave propagation in bubbly liquids that can be compared with the solutions of the full nonlinear model.

In the classical theory, it is possible to derive a wave equation for the effective pressure far from any bubble [22]

$$
\nabla^{2} P_{\infty}+k_{m}^{2} P_{\infty}=0
$$

where $P_{\infty}$ stands for the amplitude of the effective pressure wave acting far from the bubbles. The value of the effective wave number in the mixture, $k_{m}$, can be obtained as

$$
k_{m}^{2}=\frac{\omega^{2}}{c^{2}}+4 \pi \omega^{2} \int_{0}^{\infty} \frac{a f(a) d a}{\omega_{0}^{2}-\omega^{2}+2 \imath \delta \omega},
$$

where $\imath=\sqrt{-1}, \omega$ is the angular frequency, $c$ is the speed of sound in the liquid, $f(a)$ is the bubble distribution function, and $\omega_{0}$ and $\delta$ are usually identified with the bubble resonance frequency and damping constant obtained from the linearization of the Rayleigh-Plesset equation,

$$
R \ddot{R}\left(1-\frac{\dot{R}}{c}\right)+\frac{3}{2} \dot{R}^{2}\left(1-\frac{\dot{R}}{3 c}\right)=S .
$$

In this equation $S$ stands for the driving term that can be written as

$$
S=\left(1+\frac{\dot{R}}{c}+\frac{R}{c} \frac{\partial}{\partial t}\right)\left(H_{i}+\frac{\partial \Phi_{\infty}}{\partial t}\right)+I,
$$

where $H_{i}$ is the liquid enthalpy at the bubble interface $H_{i}=$ $\int_{p_{0}}^{p_{i}} \frac{d p}{\rho}, \Phi_{\infty}$ represents the potential induced by the background flow field, $\frac{\partial \Phi_{\infty}}{\partial t}=-\int_{p_{0}}^{p_{\infty}} \frac{d p}{\rho}$, and $I$ is the potential from the $N$ surrounding bubbles impacting at the location of the $i$ th bubble $\boldsymbol{x}_{i}$,

$$
I=\sum_{j \neq i}^{N} \frac{\partial \Phi_{j}\left(\boldsymbol{x}_{i}, t\right)}{\partial t} .
$$

For weak perturbations we can simplify the driving term as

$$
S=\left(H_{i}+\frac{\partial \Phi_{\infty}}{\partial t}\right)+\sum_{j \neq i}^{N} \frac{\partial \Phi_{j}\left(\boldsymbol{x}_{i}, t\right)}{\partial t},
$$

which reduces to the expression for the classical driving term when direct bubble-bubble interactions are neglected, i.e.,

$$
\lim _{\beta \rightarrow 0} S=\frac{p_{i}-p_{0}-P_{\infty} \sin (\omega t)}{\rho} .
$$


Thus, imposing a bubble radius oscillation of the form

$$
R_{i}=R_{i, 0}\left(1+X_{0} e^{\imath \omega t}\right),
$$

where $X_{0} \ll 1$ and $R_{i, 0}$ is the equilibrium bubble radius at the reference pressure $p_{0}$, the expressions for the bubble resonant frequency and damping coefficients are [22]

$$
\begin{gathered}
\omega_{0}^{2}=\frac{p_{0}}{\rho R_{i, 0}^{2}}\left(\operatorname{Re}(\Upsilon)-\frac{2 \sigma}{R_{i, 0} p_{0}}\right), \\
\delta=\frac{2 \mu}{\rho R_{i, 0}^{2}}+\frac{p_{0}}{2 \omega \rho R_{i, 0}^{2}} \operatorname{Im}(\Upsilon)+\frac{\omega^{2} R_{i, 0}}{2 c},
\end{gathered}
$$

where $\sigma$ is the surface tension, $\rho$ and $\mu$ are the liquid density and viscosity, and $\Upsilon$ is a complex valued function that relates the pressure and radius changes in the linear regime. This function is obtained from the solution of the conservation equations inside the bubble under proper boundary conditions $[23,24]$. Using the specific heat ratio $\gamma$ and the Péclet number Pe $=\omega R_{i, 0}^{2} / D_{g}, D_{g}$ being the gas thermal diffusivity, the function $\Upsilon$ can be written as

$$
\Upsilon=\frac{3 \gamma}{1-3(\gamma-1) l \mathrm{Pe}^{-1}[\sqrt{\imath \mathrm{Pe}} \operatorname{coth}(\sqrt{\imath \mathrm{Pe}})-1]} .
$$

The attenuation and phase velocity of the wave can then be obtained as a function of the complex and imaginary part of the effective velocity [22].

\section{B. Extended linear model accounting for direct bubble-bubble interactions}

The linear solutions obtained above only consider the interaction of bubbles present in the system through the averaged field. The full solution can be obtained applying the procedure proposed by Keller and Miksis [25] for a single bubble to the entire bubble cluster. In this case, we consider that the background potential $\Phi_{\infty}$ is known and we need to solve for the potentials emitted by each bubble in the cloud. When the incident field is a planar wave propagating along $x$ with angular frequency $\omega$ and pressure amplitude $P^{*}$, then,

$$
\Phi_{\infty}=-\left(P^{*} / \rho \omega\right) \cos [\omega(t-x / c)] .
$$

In this situation it is readily shown that in the linear regime, except for frequencies much higher than the natural resonance frequency, the driving term can be written as

$$
S=\frac{p_{i}-p_{0}-P^{*} \sin (\omega t)}{\rho}+I,
$$

where $P^{*}$ is constant and $I$ accounts for all the bubbles present in the cluster. Given the number of bubbles present in the system $N$ that interact directly with a given bubble, it is possible to write $N$ Bernoulli equations at the interface of each bubble present in a given volume,

$$
\sum_{j=1}^{N} \frac{\partial \Phi_{j}\left(\boldsymbol{x}_{i}, t\right)}{\partial t}=-\frac{1}{2} \dot{R}_{i}^{2}-H_{i}-\frac{\partial \Phi_{\infty}}{\partial t} .
$$

Thus, we obtain a system of $N$ equations that can be solved for the potentials $\Phi_{i}$ given the background potential variations $\frac{\partial \Phi_{\infty}}{\partial t}$. The $N$ potentials need to be evaluated at every bubble location. Assuming that the bubble is a monopolar source emitting a spherical wave decaying with the inverse of the distance between the $i$ th and $j$ th bubble, $d_{i j}$, the potential can be written as a function of the retarded time $\tau=\frac{d_{i j}}{c}$ as

$$
\Phi_{j}\left(\boldsymbol{x}_{i}, t\right)=\frac{1}{d_{i j}} f(t-\tau)=\Phi_{j}\left(t-\tau, \boldsymbol{x}_{j}\right) \frac{R_{j}(t-\tau)}{d_{i j}} .
$$

This concept has been used by Zeravcic [26] to investigate the collective response of bubble clusters. In this work this solution will be obtained using three-dimensional simulations obtained with the nonlinear model presented above in the limit of small perturbations.

The reasoning above allows one to obtain numerical solutions for the full problem but it is computationally intensive when $N$ is large. Thus, we derive here correction terms for the classical linear theory that capture the influence of the scattered waves emitted by each individual bubble. To this end, it is interesting to compare the equation used by the classical linear theory [Eqs. (5) and (11)] with that obtained from the solution of the individual potentials [Eqs. (16) and (17)]. As one can clearly see, part of the potential that is emitted by the bubbles is already accounted for by considering a coherent planar wave propagating across the bubbly media that is dissipated with a phase velocity influenced by the effective medium. Thus, if one desires to introduce a correction to account for direct bubble-bubble interactions in the classical linear theory we have to look for a correction term of the type

$$
S=\frac{p_{i}-p_{0}-P_{\infty} \sin (\omega t)}{\rho}+I^{*},
$$

where now $I^{*}$ represents the part of the potentials emitted by the surrounding bubbles not captured by the averaged field equations. The procedure to obtain the exact value of $I^{*}$ is as computationally demanding as it is to solve the full problem [Eq. (17)] and therefore it is not interesting for practical purposes. As an alternative, we can obtain an estimation of $I^{*}$ modeling the potential emitted from the $j$ th bubble using the total potential $\Phi$ at a given location,

$$
\Phi \equiv \Phi_{\infty}+\Phi_{j}+\sum_{k \neq j}^{N} \Phi_{k}
$$

The difference between the total potential and the background potential at the interface of the $j$ th bubble has to satisfy the Bernoulli equation at the interface of the $j$ th bubble:

$$
\frac{\partial}{\partial t}\left[\Phi\left(R_{j}\right)-\Phi_{\infty}\left(R_{j}\right)\right]=-\frac{1}{2} \dot{R}_{j}{ }^{2}-\frac{p_{j}-p_{\infty}}{\rho} .
$$

As previously stated, only part of this potential can be directly attributed to the $j$ th bubble,

$$
\Phi\left(R_{j}\right)-\Phi_{\infty}\left(R_{j}\right)=\Phi_{j}\left(R_{j}\right)+\sum_{k \neq j}^{N} \Phi_{k}\left(R_{j}\right)
$$

In fact, using the equation above it is possible to rewrite Eq. (22) as an equation for the potential of the $j$ th bubble as

$$
\frac{\partial \Phi_{j}\left(R_{j}\right)}{\partial t}=g\left[-\frac{1}{2} \dot{R}_{j}{ }^{2}-\frac{p_{j}-p_{\infty}}{\rho}\right],
$$


where $g$ is defined as

$$
g \equiv \frac{\frac{\partial}{\partial t} \Phi_{j}\left(R_{j}\right)}{\frac{\partial}{\partial t}\left(\Phi_{j}\left(R_{j}\right)+\sum_{k \neq j}^{N} \Phi_{k}\left(R_{j}\right)\right)} .
$$

This function, bounded between zero and one, is unknown for a general case. In the limiting case where the potentials emitted by the surrounding bubbles are more important than the potential generated by the bubble itself, the function becomes zero indicating that direct bubble-bubble interactions are not relevant. On the contrary, when the sum of the potentials emitted by the surrounding bubbles is negligible, $g$ becomes unity, e.g., there is no need to solve for the system of $N$ equations to obtain the potential emitted by each bubble because the coupling effect is negligible.

The relative importance of the potentials emitted by the surrounding bubbles can be characterized in terms of the attenuation induced by the effective medium surrounding the bubble. To compute the total interaction potential impacting the $i$ th bubble we integrate the contribution of all potentials emitted at a distance smaller than $L=C R_{0} / \beta_{0}^{1 / 3}$, where $C$ is a free parameter to be determined later. At distances larger than $L$, the bubbles are assumed to interact through the averaged field. In the absence of attenuation we can write

$$
\sum_{j=1}^{N} \frac{\partial \Phi_{j}\left(\boldsymbol{x}_{i}, t\right)}{\partial t} \approx \int_{0}^{L} \frac{R_{0}}{r} \frac{\partial \Phi_{j}\left(\boldsymbol{x}_{j}, t\right)}{\partial t} 4 \pi r^{2} n d r
$$

where $n$ is the number of bubbles per unit volume that can be obtained as

$$
n=\frac{\beta_{0}}{4 / 3 \pi R_{0}^{3}}
$$

Equation (26) is also expected to be applicable in a general case for small distances, where the attenuation of the effective medium is negligible. However, if $L$ takes large values, the effective medium is going to induce an attenuation rate $A$ for the effective contribution of bubbles far away from the $i$ th bubble. If $A$ is known, the effective contribution of the potentials is corrected

$$
\sum_{j=1}^{N} \frac{\partial \Phi_{j}\left(\boldsymbol{x}_{i}, t\right)}{\partial t} \approx \int_{0}^{L} \frac{R_{0}}{r} \frac{\partial \Phi_{j}\left(\boldsymbol{x}_{j}, t\right)}{\partial t} 4 \pi r^{2} n e^{-A r} d r
$$

and the direct bubble-bubble interaction term correction can then be obtained as

$$
I^{*}=\sum_{j \neq i}^{N} \frac{\partial \Phi_{j}\left(\boldsymbol{x}_{i}, t\right)}{\partial t}=\sum_{j=1}^{N} \frac{\partial \Phi_{j}\left(\boldsymbol{x}_{i}, t\right)}{\partial t}-\frac{\partial \Phi_{i}\left(\boldsymbol{x}_{i}, t\right)}{\partial t} .
$$

To illustrate the influence of bubble-bubble interactions, let us now consider an ideal situation where all the bubbles emit the same potential intensity at a given instant (e.g., all the bubbles have the same pressure and expansion velocity at a given instant). Thus, we write Eq. (24) as

$$
\frac{\partial \Phi_{j}\left(R_{j}\right)}{\partial t}=g P_{0}
$$

In this case, for distances smaller than $L \omega / c<1$, Eq. (28) can be exactly evaluated as

$$
\sum_{j=1}^{N} \frac{\partial \Phi_{j}\left(\boldsymbol{x}_{i}, t\right)}{\partial t}=\frac{3\left(e^{A L}-A L-1\right) \beta_{0}}{\left(A R_{0}\right)^{2} e^{A L}+3\left(e^{A L}-A L-1\right) \beta_{0}} P_{0} .
$$

When $L$ is large $(A L \gg 1)$, we can approximate the sum of all the potentials emitted by the bubbles at a given location,

$$
\sum_{j=1}^{N} \frac{\partial \Phi_{j}\left(\boldsymbol{x}_{i}, t\right)}{\partial t} \approx \frac{3 \beta_{0}}{\left(R_{0} A\right)^{2}+3 \beta_{0}} P_{0}
$$

and the interaction correction term can be obtained using Eq. (29) as

$$
I^{*}=\frac{-\left(R_{0} A\right)^{2}}{\left(R_{0} A\right)^{2}+3 \beta_{0}} P_{0} .
$$

This equation reveals that the interaction term neglected in the classical theory can be expressed in terms of the effective attenuation of the medium. If the effective attenuation at a given frequency is below a critical attenuation $A_{c}$ value,

$$
A_{c}=\frac{\sqrt{3 \beta_{0}}}{R_{0}},
$$

then $I^{*}$ tends to zero when decreasing the attenuation. For dilute systems, where the attenuation is proportional to the void fraction for frequencies well below the bubble resonance frequency [22], the result above implies that the term neglected in the classical approach is of order $O\left(\beta_{0}\right)$. When the attenuation is large, the magnitude of the total interaction potential is maximized $\left(\left|I^{*}\right| \rightarrow P_{0}\right)$ tending to oppose a significant resistance to the expansion/compression of the bubbles present in the system. In this case it is important to note that, as the effective medium induces a large attenuation on the potentials emitted by the surrounding bubbles, the contribution of bubbles further than few times the averaged interbubble distance is going to be negligible. Thus, Eq. (26) is more suitable to obtain the interaction potentials at small distances. In this case the potential emitted by the $j$ th bubble impacting on the $i$ th bubble at distance $r$ can be obtained introducing the time lag, $\tau=\frac{d_{i j}}{c}, R_{j}=R_{0}\left(1+X_{0} e^{\imath \omega t} e^{-\imath \omega \tau}\right)$ as

$$
\frac{\partial \Phi_{j}\left(\boldsymbol{x}_{i}, t\right)}{\partial t}=\frac{g}{\rho}\left(p_{g e} \Upsilon-\frac{2 \sigma}{R_{0}}+4 \mu \iota \omega\right) X_{0} e^{-l \omega \tau} \frac{R_{0}}{r} .
$$

For monodisperse mixtures we replace Eq. (35) with Eq. (26) to integrate it from zero to a given distance $L$. Beyond this distance bubbles are assumed to interact through the averaged field. Thus we obtain

$$
I^{*}=I_{0} X_{0} e^{\imath \omega t},
$$

where $I_{0}$ is zero in the regions of low attenuation $\left(A<A_{c}\right)$ and it becomes

$$
\begin{aligned}
I_{0}= & -\frac{1}{\rho} \frac{3 \beta_{0}}{\left(R_{0} \omega / c\right)^{2}}\left[1-e^{-\imath L \omega / c}(1+\imath L \omega / c)\right] \\
& \times\left(p_{g e} \Upsilon-\frac{2 \sigma}{R_{0}}+4 \mu \iota \omega\right),
\end{aligned}
$$


above the critical attenuation [Eq. (34)]. Following the same procedure used in the classical linear theory, the modification introduced in the driving term of the Rayleigh-Plesset equation due to direct bubble-bubble interactions gives an additional correction on the bubble's resonance frequency and damping coefficient as

$$
\begin{gathered}
\omega_{0}^{2}=\frac{p_{0}}{\rho R_{i, 0}^{2}}\left(\operatorname{Re}(\Upsilon)-\frac{2 \sigma}{R_{i, 0} p_{0}}\right)+\frac{\operatorname{Re}\left(I_{0}\right)}{R_{i, 0}^{2}}, \\
\delta=\frac{2 \mu}{\rho R_{i, 0}^{2}}+\frac{p_{0}}{2 \omega \rho R_{i, 0}^{2}} \operatorname{Im}(\Upsilon)+\frac{\omega^{2} R_{i, 0}}{2 c}+\frac{\operatorname{Im}\left(I_{0}\right)}{2 \omega R_{i, 0}^{2}} .
\end{gathered}
$$

As stated above, this expression is not closed. It contains an unknown free parameter $C$ and a frequency-dependent function $g$ that is bounded between zero (for regimes with small attenuation) and one (for regimes with large attenuation). We note that $C$ can be identified with a nondimensional interaction distance and therefore we expect it to be approximately constant irrespective of the bubble radius and void fraction $C=\frac{L \beta^{1 / 3}}{R_{0}}$. Numerical tests, which solve for the full problem, are used in the next section to obtain a better understanding about reasonable values of $C$ and $g$ for a general case.

In the next section we provide numerical and experimental evidence that supports the veracity of the arguments exposed in this section.

\section{NUMERICAL TESTS}

We now describe the general simulation setup that is used to solve the full (nonlinear) bubble model. We consider air bubbles in water with a known concentration $\beta_{0}$ and an average radius $R_{0}$ defined at a reference pressure, $p_{0}=1 \mathrm{~atm}$, and temperature, $T_{0}=25^{\circ} \mathrm{C}$. The bubbles are seeded according to a known probability distribution function $f(a)$ that represents the distribution of equilibrium bubble radii in the system. For instance, for the polydisperse case included in this paper, we initialize the bubble population with a random distribution of bubbles obeying the following Gaussian distribution:

$$
f(a)= \begin{cases}\frac{1}{b \sqrt{2 \pi}} \exp \left[\frac{-\left(a-R_{0}\right)^{2}}{2 b^{2}}\right], & 0.1 R_{0} \leqslant a \leqslant 5 R_{0} \\ 0, & \text { otherwise, }\end{cases}
$$

where $R_{0}=110 \mu \mathrm{m}$ and $b / R_{0}=0.25$ (Fig. 1). Bubbles are distributed randomly in a rectangular domain of dimensions $\left[-L_{x} / R_{0}: L_{x} / R_{0}\right]\left[-L_{y} / R_{0}: L_{y} / R_{0}\right]\left[-L_{z} / R_{0}: L_{z} / R_{0}\right]$.

A one-dimensional Gaussian pressure pulse is initialized in a bubbly liquid at rest $(\boldsymbol{u}=0)$ following the equation

$$
p(x)=p_{0}\left(1+\epsilon \exp \left[-x^{2} / s_{p}^{2}\right]\right) .
$$

The initial bubble radius is then obtained from Laplace's equation so that bubbles are in equilibrium with the initial pressure. This unphysical initialization procedure is only an artifact that only influences the initial state of bubbles in a small region with a characteristic length of the order of $s_{p}$, and has no significant impact on the results. For the simulation contained in this work, we have set $\epsilon=10^{-4}$ and $\frac{s_{p}}{R_{0}}=1$.

To obtain a representative behavior of the averaged bubble propagation, the pressure disturbance is integrated in a plane

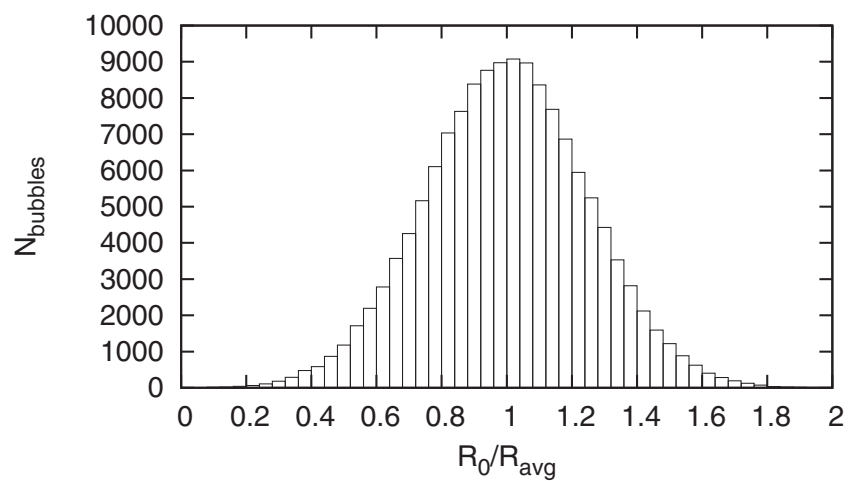

FIG. 1. Histogram of the bubble size distribution used in the simulation for validation against the classical linear theory results (results presented in Fig. 2). $R_{0}=110 \mu \mathrm{m}$.

perpendicular to the wave propagation. Thus, we define the pressure perturbation $p^{\prime}$ at a given sampling point $x_{i}$ as

$$
p^{\prime}\left(x_{i}\right)=\frac{\iint\left[p\left(x_{i}, y, z\right)-p_{0}\right] d y d z}{\iint d y d z} .
$$

Then, following Ref. [27], the attenuation and speed of sound are obtained using the Fourier transform of the temporal evolution of $p^{\prime}\left(x_{i}\right)$ at multiple sampling points. The values for the complex and real parts of the complex wave number are fitted by least squares using the data from five equally spaced sampling points. In particular, two different sets are used; the first ones use a spacing of $10 R_{0}$ chosen to correctly capture the speed of sound in the high frequency range. The second set of points is chosen with spacing $30 R_{0}$ to minimize the influence of round-off errors in the low attenuation regions.

\section{A. Validation test case: Wave propagation results neglecting direct bubble-bubble interactions}

One of the advantages of the numerical approach is that it allows us to reproduce ideal situations where the code can be rigorously validated against traditional linear theories. In particular, in this section we will switch off the direct bubblebubble interaction term and use a coarse mesh in the $z$ direction so that the concentration in every computational cell is given by the averaged concentration. In these conditions, the full model presented in Ref. [18] is expected to recover the classical linear theory predictions for the attenuation and phase speed of linear waves propagating across a bubbly liquid neglecting direct bubble-bubble interactions.

The computational domain size is set to $\left(\frac{L_{x}}{R_{0}}\right)\left(\frac{L_{y}}{R_{0}}\right)\left(\frac{L_{z}}{R_{0}}\right) \in$ $[-3500: 3500][-250: 250][-250: 250]$, the grid size is $\left(\Delta x / R_{0}\right)\left(\Delta y / R_{0}\right)\left(\Delta z / R_{0}\right)=10 \times 10 \times 500$, and the initial void fraction, $\beta_{0}=\frac{4}{3} \pi n \int a^{3} f(a) d a$ is set to $\beta_{0}=$ $2.5 \times 10^{-4}$.

As expected, Fig. 2 shows good agreement between the theoretical predictions and the simulation results, validating the capability of the code to reproduce the theoretical results predicted by the classical linear theory, in those conditions where the grid does not resolve the scattered waves emitted by the individual bubbles. Both attenuation and phase velocity are very well reproduced for frequencies up to the resonance 

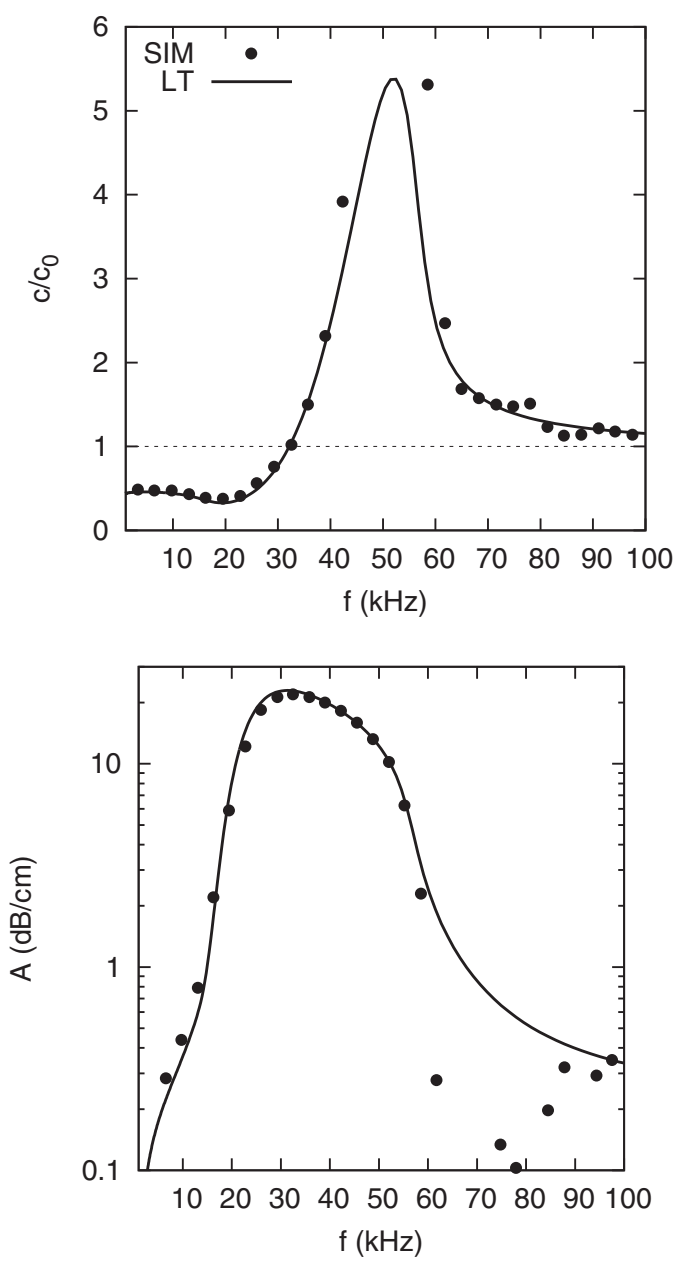

FIG. 2. Speed of sound and attenuation of a polydisperse bubble cloud of randomly distributed bubbles obtained from simulation results and comparison with the traditional linear theory neglecting bubble-bubble interactions [22].

frequency. The peak on the phase velocity is especially challenging for the numerical method due to the large velocities obtained there, but the match for the attenuation for values above $0.2 \mathrm{~dB} / \mathrm{cm}$ is remarkably good. The results obtained become noisy as we approach the maximum frequency that can be numerically solved, which is defined as $f_{\max }=c / \Delta x$. For the simulations included in this work we set $\Delta x=10 R_{0}$. When comparing the maximum frequency with the bubble natural frequency $\left(f_{N}=\frac{1}{2 \pi} \sqrt{\frac{3 \gamma p_{0}}{\rho R_{0}^{2}}}\right)$ we conclude that, for air and water systems at atmospheric pressure, we expect the results to be highly influenced by spatial discretization errors for nondimensional frequencies of the order of ten times the bubble resonance frequency $\left(\frac{f}{f_{N}} \approx 10\right)$.

\section{B. Numerical and theoretical results of wave propagation accounting for direct bubble-bubble interactions}

In order to investigate the influence of direct bubblebubble interactions on wave propagation we consider various monodisperse bubble clouds with a different concentration $\beta_{0}$ and bubble radius $R_{0}$. Unlike the previous case, the grid now is refined in order to capture not only the coherent
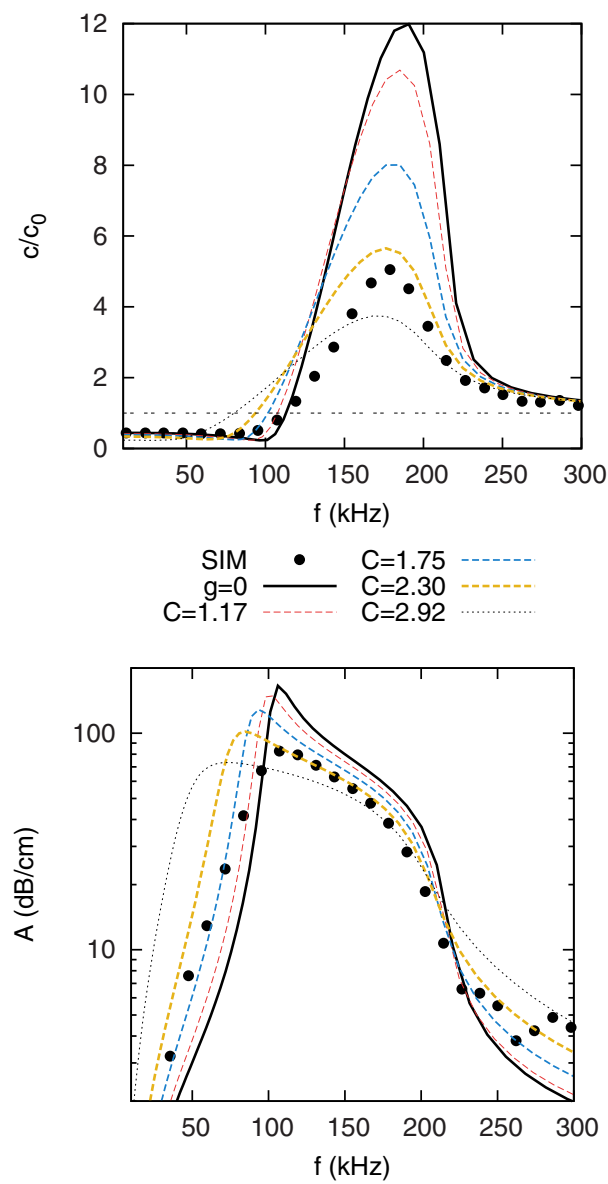

FIG. 3. (Color online) Influence of the fitting parameter $L / R_{0}$ on the phase velocity and attenuation of a monodisperse bubble cloud of randomly distributed air bubbles in water obtained from simulation results for case A ( $g=1$ in all cases). For reference, the predictions of the classical linear theory $(g=0)$ and the results from simulations are included.

wave, but also the waves scattered by the surrounding bubbles that are a result of direct bubble-bubble interaction. The simulation domain, $\left(\frac{L_{x}}{R_{\mathrm{ref}}}\right)\left(\frac{L_{y}}{R_{\mathrm{ref}}}\right)\left(\frac{L_{z}}{R_{\mathrm{ref}}}\right) \in[-3500: 3500][-250$ : $250][-250: 250]$, is discretized with a regular Cartesian grid of size $\Delta x / R_{\text {ref }}=\Delta y / R_{\text {ref }}=\Delta z / R_{\text {ref }}=10$, where $R_{\text {ref }}=$ $30 \mu \mathrm{m}$. The bubble number density is initially set to a constant value given by $n_{\text {ref }}=\frac{\beta_{\text {ref }}}{4 / 3 \pi R_{\text {ref }}^{3}}$ where $\beta_{\text {ref }}=2 \times 10^{-4}$. Monodisperse bubble clouds with $R_{0}<R_{\text {ref }}$ at constant $n_{\text {ref }}$ fulfill two different conditions: The bubble radius remains small compared to the grid size and the interspacing between bubbles is larger than the grid size. The grid spacing quoted above and used in the calculations that follow was selected

TABLE I. Bubble radius, concentration, and best fitting interaction distance $L$ for the simulation results shown in Fig. 4.

\begin{tabular}{lccrr}
\hline \hline & $R_{0}(\mu \mathrm{m})$ & $f_{\text {res }}(\mathrm{kHz})$ & \multicolumn{1}{c}{$\beta_{0}$} & \multicolumn{1}{c}{$L / R_{0}$} \\
\hline Case A & 30 & 109 & $2 \times 10^{-4}$ & 40 \\
Case B & 15 & 219 & $2.5 \times 10^{-5}$ & 63 \\
Case C & 7.5 & 438 & $3.125 \times 10^{-6}$ & 133 \\
\hline \hline
\end{tabular}



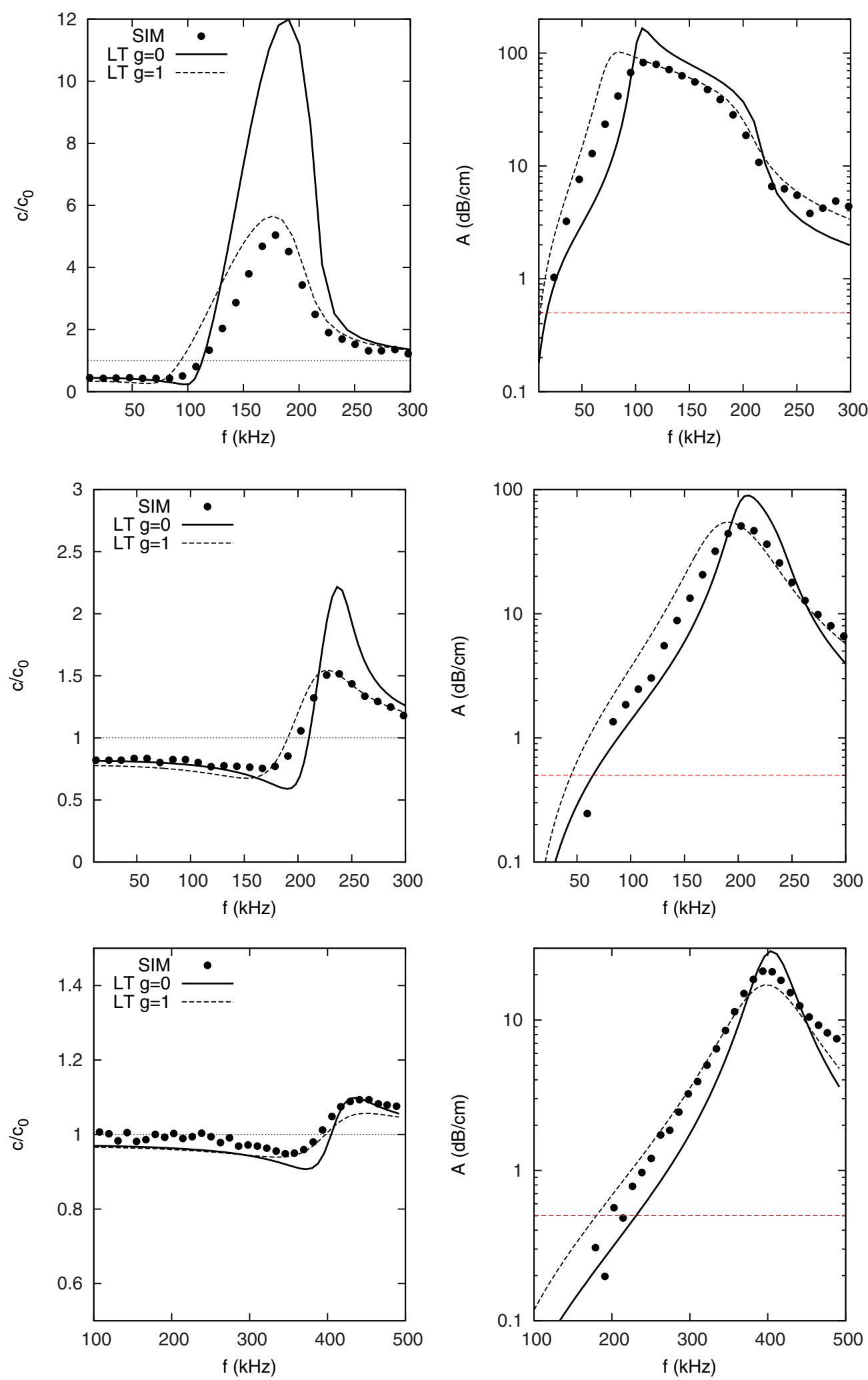

FIG. 4. (Color online) Phase velocity and attenuation of a monodisperse bubble cloud of randomly distributed air bubbles in water obtained from simulation results for cases A (top), B (middle), and C (bottom). The dashed horizontal line on the attenuation plots indicates the upper bound for which the numerical attenuation values are reliable, due to numerical dissipation. For reference, the predictions of the classical linear theory and the proposed extension of the theoretical results with constant $C=2.3$ are included. 
by examining results on a series of progressively finer meshes and verifying an approximate independence of the phase speed and attenuation to grid resolution. As was reported in [18], this establishes that we are adequately capturing the local pressure and velocity fluctuations induced by the bubbles. As the leading-order truncation error in our WENO scheme is dissipative, we also computed attenuation rates for waves propagating on the finest grid in the absence of bubbles, i.e., a pure inviscid liquid. As numerical dissipation is the only source of attenuation in this case, this serves to establish a lower bound on the attenuation rates that we can reliably assess. The value is indicated on the attenuation plots that follow.

Figure 3 contains the phase speed and attenuation simulation data obtained from simulations for the conditions of case A (Table I). Theoretical predictions for the classical theory (LT $g=0$ ) and the extended theory with $g=1$ (LT $g=1$ ) for different values of $C$ are included for reference. Recall that we expect the real solution to be bounded between these two limiting cases, where results tend to the classical linear theory predictions for the regions with low attenuation $\left(A \ll A_{c}=8.11 \mathrm{~dB} / \mathrm{cm}\right)$ whereas the predictions for $g=1$ should better represent the regions of large attenuation $(A>$ $A_{c}$ ). The solutions in the limit of $g=0$ are independent of the nondimensional interaction distance $C$. On the contrary, when $g>0$, results are sensitive to $C$. Based on this fact, we use the region with the largest attenuation to calibrate the nondimensional interaction distance $C$. We remark that in this region we expect $g$ to be approximately constant and equal to 1 . The peak values of both phase speed and attenuation are overpredicted by the linear theory up to factor 2 for case A. The modified linear theory significantly improves the fitting between theory and numerical results fitting the "nondimensional interaction distance" to $C=2.3$. This value falls within the range of the averaged interspacing, which is consistent with the fact that only bubbles nearby directly interact with a given bubble, whereas the rest interact through the averaged field.

Figure 4 depicts the results for the various conditions included in Table I, all of them with constant bubble number density. We can see how the differences between the two limiting cases become clear as the concentration increases for frequencies of the order of the bubble natural resonance frequency. As expected, direct bubble-bubble interactions become less important as void fraction decreases. The maximum errors are significantly reduced down to $20 \%$ for concentrations of the order of $10^{-5}$ and the differences are almost negligible for lower concentrations. The critical concentration threshold for which direct bubble-bubble interactions are important therefore coincides which the expected value $(\beta=$ $10^{-4}$ ) although one must keep in mind that we only observe significant differences for frequencies of the order of the resonance frequency. Finally, the distance required to obtain the best fitting value increases as the void fraction decreases (Table I). This is consistent with the fact that as concentration decreases, the bubble interspacing increases. A constant value of $C=2.3$ in the three cases significantly improves the fitting between the extended linear theory and numerical simulations in the range of frequencies where the attenuation is above the critical value $A_{c}$.

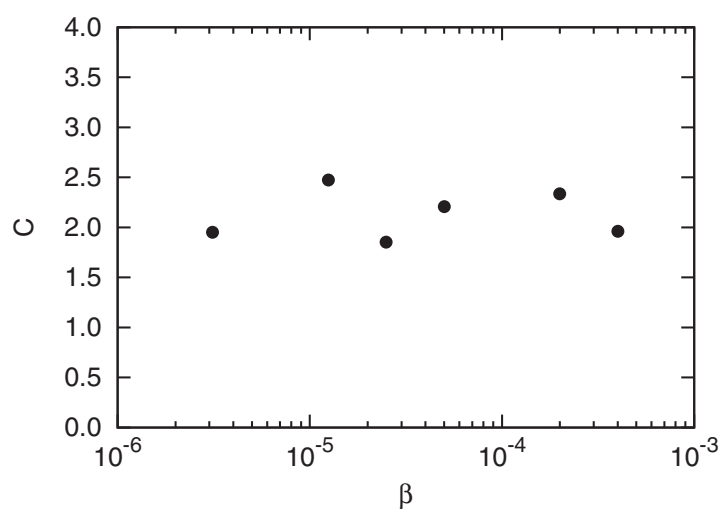

FIG. 5. Best fitting value of the nondimensional interaction distance, $L \beta^{1 / 3} / R_{0}$, for various bubble radius and concentrations for monodisperse bubble clouds. The number of bubbles range from $n / n_{\text {ref }}=0.5$ to 2 . The nondimensional value $C=\frac{L \beta^{1 / 3}}{R_{0}}$ remains approximately constant irrespective of the concentration and bubble radius.

To gain more insight into the applicability of the fitting constant value to a wider range of conditions we represent in Fig. 5 the nondimensional fitting parameter $C=\frac{L \beta^{1 / 3}}{R_{0}}$ for various tests with slightly higher and lower values of the bubble number density. Remarkably, the value of $C$ remains approximately constant ranging from 2 to 2.5 for all the different conditions tested, which include a very large range of bubble concentrations.

It is interesting to note that some of the observations extracted from Fig. 4 have been systematically observed in previous experimental and theoretical studies reported in the literature. For instance, Silberman [9] has observed a systematic overprediction of attenuation predicted by the traditional linear theory on the regions of large attenuation above the resonance frequency, slightly underpredicting the attenuation coefficient for frequencies below the attenuation peak. Similar mismatching has been found by Commander and Prosperetti in Ref. [22] when fitting the data from both Silberman [9] and Fox et al. [8]. This later case is interesting because measurements also include phase speeds experimentally measured in the frequency range where there is a clear mismatch in the attenuation. The theoretical results derived by Sangani also pointed out that the attenuation around the resonance frequency is lower than that predicted by the classical theory when introducing correction terms to account for the effect of non-negligible void fractions [6]. Wilson et al. [11] and Leroy et al. [12] report lower values of the phase velocities than those predicted by the traditional linear theory in the range of frequencies where we find large attenuation. These observations are consistent with the numerical results obtained in this work and with the fact that direct bubble-bubble interactions are probably negligible in the low frequency limit (e.g., $g \approx 0$ ), whereas they have an important influence for frequencies above the resonance frequency.

In an attempt to reproduce this effect observed experimentally, we numerically reproduce the lowest concentration tested by Silberman [9] $\left(\beta_{0}=3.77 \times 10^{-4}\right)$, where the bubble size 

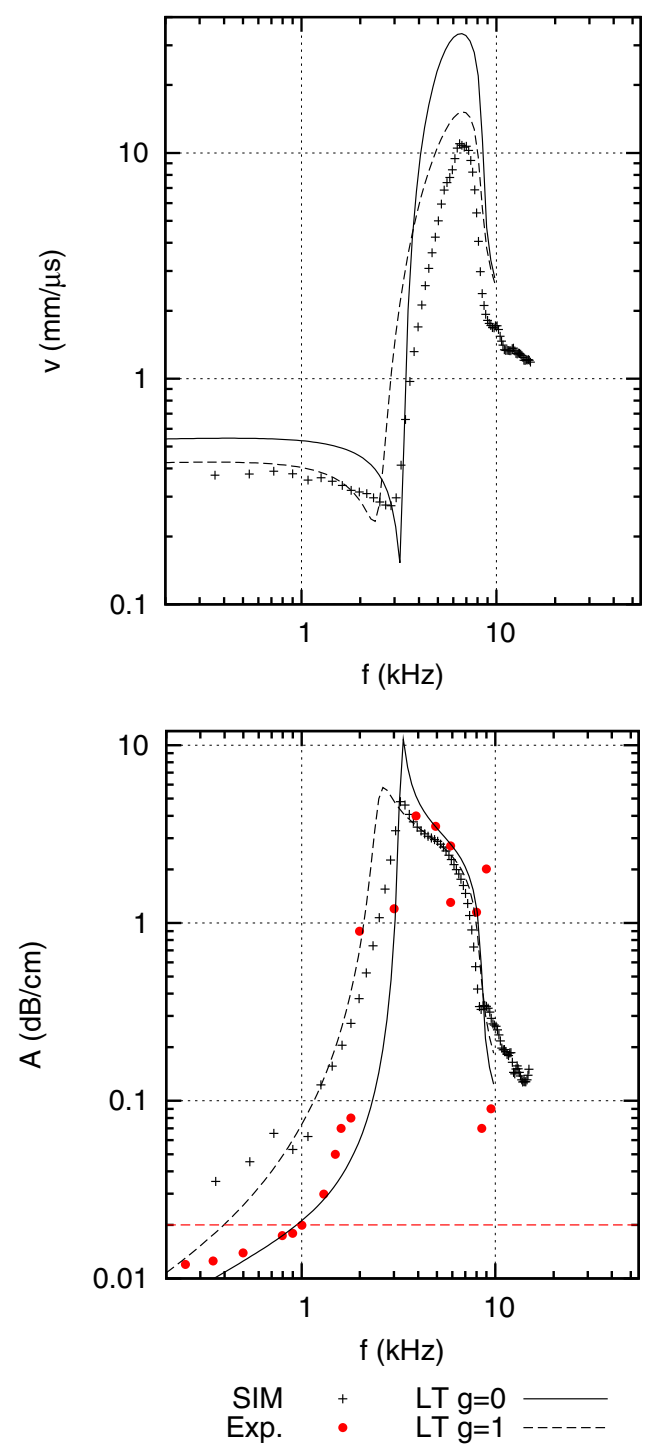

FIG. 6. (Color online) Phase velocity and attenuation of a polydisperse bubble cloud of randomly distributed bubbles obtained from simulation results and comparison with the experimental results of Silberman [9], the linear theory (LT) predictions of Ref. [22] (LT $g=0$ ) and the modified linear theory accounting for direct bubble-bubble interactions in the limiting case of $g=1$. The dashed horizontal line on the attenuation plots indicates the upper bound for which the numerical attenuation values are reliable, due to numerical dissipation.

ranges between 0.994 and $1.07 \mathrm{~mm}$. These conditions are in the limit of the highest values of void fraction for which it is possible to obtain a grid independent solution with the

[1] L. Van Wijngaarden, J. Fluid Mech. 33, 465 (1968).

[2] M. Ainslie and T. Leighton, J. Acoust. Soc. Am. 130, 3184 (2011).

[3] R. Chapman and M. Plesset, J. Basic Eng. (Trans. ASME) 93, 373 (1971).

[4] A. Prosperetti, J. Acoust. Soc. Am. 61, 17 (1977). current numerical model. In Fig. 6 we include the theoretical predictions for the two limiting cases corresponding to the classical linear theory $(g=0)$ and the limit of $g=1$, for which we use the value of $L$ previously fitted from numerical results $\left(L \beta^{1 / 3} / R_{0} \approx 2.3\right)$. The agreement between the modified linear theory, numerical simulations, and experiments is relatively good in the regions of large attenuation, where the classical linear theory overpredicts the attenuation. At the lowest frequencies, where the attenuation is small, the experimental values tend to the $g=0$ solution, consistent with the previous discussion. We note that the simulation results are not reliable in this regime because the attenuation values are below our estimate for the numerical dissipation, even on the finest mesh we considered. While finer meshes (or less dissipative schemes) could be used to alleviate this problem, we refrain from such calculations since they would not affect the conclusions drawn.

\section{v. CONCLUSIONS}

This work uses the model proposed by Fuster and Colonius [18] to study the influence of direct bubble-bubble interactions on the acoustic properties of the effective medium. Taking advantage of the results obtained with the full nonlinear model that accounts for bubble-bubble interactions, we are able to isolate the influence in the linear regime of waves scattered by individual bubbles on the effective linear acoustic response of the mixture. Consistent with previously reported experimental [9] and theoretical data [6], the numerical results provide evidence that the classical linear theory overpredicts the attenuation and phase velocity above the natural resonance frequency.

A modification of the linear theory is suggested to try to capture these effects. The new theory introduces a characteristic distance $L$ that we identify with an "interaction distance." In those regions where the attenuation is larger than a critical value defined as $A_{c}=\sqrt{3 \beta_{0}} / R_{0}$, the individual bubble response is assumed to be influenced by the waves scattered by bubbles at distances smaller than $L$, whereas the interaction with bubbles placed further takes place through the averaged pressure field. The comparison between the numerical results obtained with the full nonlinear model and the extended linear theory reveals that the proposed correction term significantly improves the theoretical predictions when the nondimensional value for the interaction distance, $L \beta_{0}^{1 / 3} / R_{0}$, is approximately constant $\left(L \beta_{0}^{1 / 3} / R_{0} \approx 2.3\right)$ for void fractions below $10^{-3}$.

\section{ACKNOWLEDGMENTS}

This work was supported by the French National Agency for Research (ANR-113S09-007-03 ) project DiAMAN.
[5] K. Ando, T. Colonius, and C. E. Brennen, J. Acoust. Soc. Am. 126, EL69 (2009).

[6] A. S. Sangani, J. Fluid Mech. 232, 221 (1991).

[7] D. Zhang and A. Prosperetti, Int. J. Multiphase. Flow 23, 425 (1997).

[8] F. Fox, S. Curley, and G. Larson, J. Acoust. Soc. Am. 27, 534 (1955). 
[9] E. Silberman, J. Acoust. Soc. Am. 29, 8 (1957).

[10] S. A. Cheyne, C. T. Stebbings, and R. A. Roy, J. Acoust. Soc. Am. 97, 1621 (1995).

[11] P. S. Wilson, R. A. Roy, and W. M. Carey, J. Acoust. Soc. Am. 117, 1895 (2005).

[12] V. Leroy, A. Strybulevych, J. Page, and M. Scanlon, J. Acoust. Soc. Am. 123, 1931 (2008).

[13] T. Leighton, P. White, C. Morfey, J. Clarke, G. Heald, H. Dumbrell, and K. Holland, J. Acoust. Soc. Am. 112, 1366 (2002).

[14] D. Farmer, S. Vagle, and D. Booth, J. Acoust. Soc. Am. 118, 2954 (2005).

[15] V. Duro, D. Rajaona, D. Decultot, and G. Maze, IEEE J. Oceanic Eng. 36, 114 (2011).

[16] D. Fuster, G. Hauke, and C. Dopazo, J. Acoust. Soc. Am. 128 5 (2010).

[17] A. Preston, T. Colonius, and C. Brennen, Phys. Fluids 19, 123302 (2007).
[18] D. Fuster and T. Colonius, J. Fluid Mech. 688, 253 (2011).

[19] E. Johnsen and T. Colonius, J. Comput. Phys. 219, 715 (2006).

[20] Y. Ilinskii, M. Hamilton, and E. Zabolotskaya, J. Acoust. Soc. Am. 121, 786 (2007).

[21] T. Colonius and D. Fuster, in Proceedings of the 8th International Symposium on Cavitation (CAV2012), edited by C.-D. Ohl, E. Klaseboer, S. W. Ohl, S. W. Gong, and B. C. Khoo (Research Publishing Services, Singapore, 2012), pp. 26-30.

[22] K. Commander and A. Prosperetti, J. Acoust. Soc. Am. 85, 732 (1989).

[23] A. Prosperetti, Ultrasonics 22, 69 (1984).

[24] A. Prosperetti, L. Crum, and K. Commander, J. Acoust. Soc. Am. 83, 502 (1988).

[25] J. Keller and M. Miksis, J. Acoust. Soc. Am. 68, 628 (1980).

[26] Z. Zeravcic, D. Lohse, and W. Van Saarloos, J. Fluid Mech. 680, 114 (2011).

[27] K. Ando, Ph.D. thesis, California Institute of Technology, 2010; see also URL http://thesis.library.caltech.edu/5859/. 